Florida International University

FIU Digital Commons

FIU Electronic Theses and Dissertations

University Graduate School

$11-13-2019$

\title{
Synergistic Activation of Inspiratory Muscles by an Adaptive Closed-loop Controller
}

\author{
Rabeya Zinnat Adury \\ radur001@fiu.edu
}

Follow this and additional works at: https://digitalcommons.fiu.edu/etd

Part of the Bioelectrical and Neuroengineering Commons

\section{Recommended Citation \\ Adury, Rabeya Zinnat, "Synergistic Activation of Inspiratory Muscles by an Adaptive Closed-loop Controller" (2019). FIU Electronic Theses and Dissertations. 4318. \\ https://digitalcommons.fiu.edu/etd/4318 \\ This work is brought to you for free and open access by the University Graduate School at FIU Digital Commons. It has been accepted for inclusion in FIU Electronic Theses and Dissertations by an authorized administrator of FIU Digital Commons. For more information, please contact dcc@fiu.edu.}




\section{FLORIDA INTERNATIONAL UNIVERSITY}

Miami, Florida

\section{SYNERGISTIC ACTIVATION OF INSPIRATORY MUSCLES BY AN ADAPTIVE CLOSED-LOOP NEUROMORPHIC CONTROLLER}

A thesis submitted in partial fulfillment of

the requirements for the degree of

MASTER OF SCIENCE

in

BIOMEDICAL ENGINEERING

by

Rabeya Zinnat Adury

2019 
To: Dean John Leonidas Volakis

College of Engineering

This thesis, written by Rabeya Zinnat Adury, and entitled Synergistic Activation of Inspiratory Muscles by an Adaptive Closed-loop Neuromorphic Controller, having been approved in respect to style and intellectual content, is referred to you for judgement.

We have read this dissertation and recommend that it be approved.

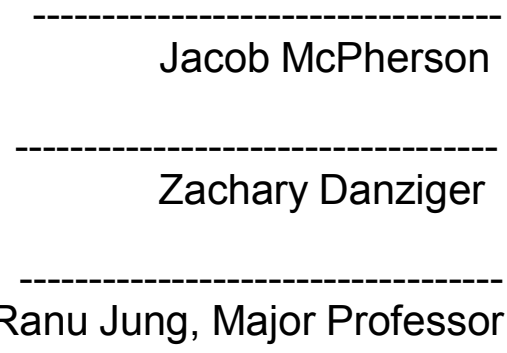

Date of Defense: November 13, 2019

Ranu Jung, Major Professor

The thesis of Rabeya Zinnat Adury is approved.

Dean John Leonidas Volakis

College of Engineering

Andrés G. Gil

Vice President for Research and Economic Development and Dean of the University Graduate School

Florida International University, 2019 


\section{DEDICATION}

I dedicate this thesis to my elder sister Runi, who had been the guardian angel to me. 


\section{ACKNOWLEDGMENTS}

I would like to express my sincere gratitude to my supervisor Dr. Ranu Jung for her valuable guidance throughout the time, without her encouragement and trust in me, I wouldn't be able to pursue my Master's degree. My heartiest gratitude to my mentor, Dr. Ricardo Siu who was patient with me and taught me and guided me the whole time. I would like to thank my committee members Dr. Jacob McPherson and Dr. Zachary Danziger for all their support. I am thankful to iian Black and Jefferson Gomes, for their loving inspiration, encouragement and suggestions. I would like to thank all the ANS lab members, I am proud to be a part of this family.

Finally, I would like to thank my parents and my sisters for their love and caring. Specially, I am thankful to my husband Polit; nobody has been more supporting than you. 


\section{ABSTRACT OF THE THESIS \\ SYNERGISTIC ACTIVATION OF INSPIRATORY MUSCLES BY AN ADAPTIVE \\ CLOSED-LOOP NEUROMORPHIC CONTROLLER,}

by

Rabeya Zinnat Adury

Florida International University, 2016

Miami, Florida

\section{Professor Ranu Jung, Major Professor}

During diaphragmatic pacing reverse recruitment of muscle fibers causes muscle fatigue. Current diaphragmatic pacing paradigms also do not permit sigh-like behavior. In spontaneously breathing anesthetized rats, studies were carried out to determine if synergized pacing of external intercostal muscles and the diaphragm would increase the efficiency of diaphragm stimulation and thus a) achieve the desired ventilatory breath volume profile with reduced diaphragm muscle fatigue, and/or b) be able to elicit sigh-like behavior by periodically increasing stimulation. Under combined inspiratory muscle stimulation, a fatigue index calculated for the diaphragm muscle ( $n=6$ rats) was significantly lower $(p<0.05)$ than in the diaphragm muscle alone. Significantly higher tidal volumes $(n=7)$ could be generated during cycles in which sigh was induced $(p<0.05)$. Thus, synergized activation of the inspiratory muscles could be used as a suitable strategy to reduce the stimulation-induced muscle fatigue and to induce a sigh-like behavior that could lead to improved respiratory health. 
CHAPTER PAGE

INTRODUCTION

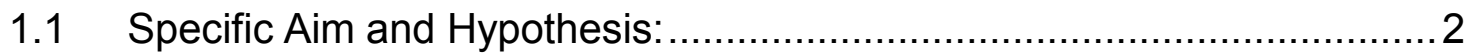

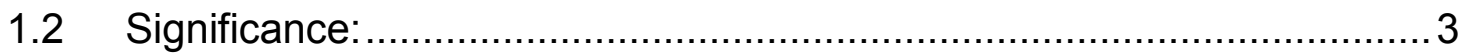

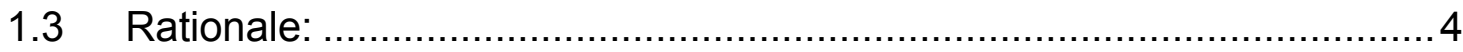

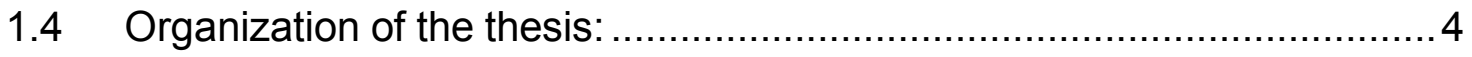

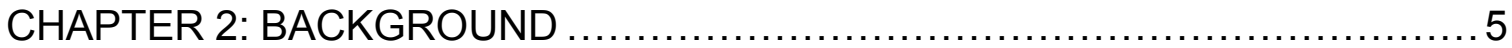

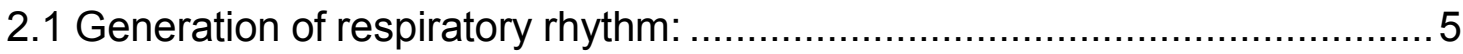

2.2 Motor unit recruitment of respiratory muscles:.......................................... 8

2.3 Techniques to restore impaired ventilation:.............................. 8

2.4 An Adaptive ventilation control system: .............................................. 12

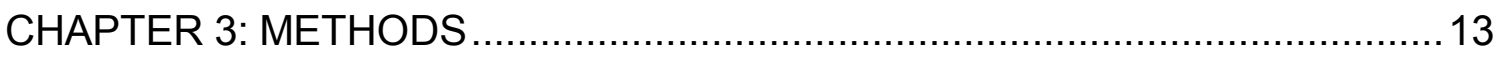

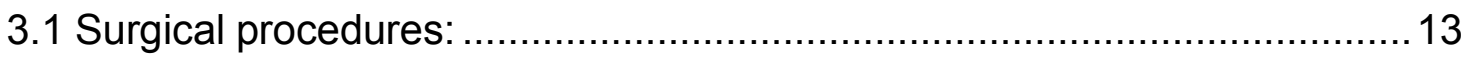

3.2 Determining pulse width duration of stimulation for the inspiratory muscle

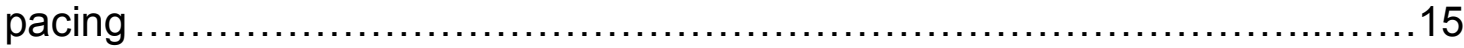

3.3 Diaphragm, external intercostal muscle pacing .................................. 15

3.4 Experimental protocol .................................................................. 16

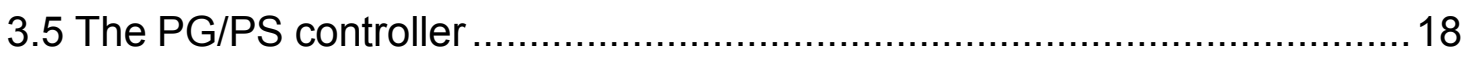

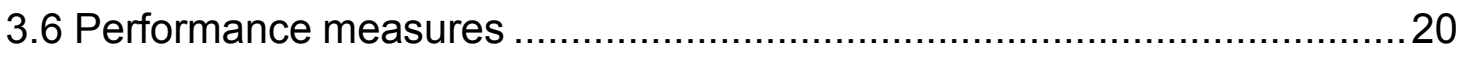

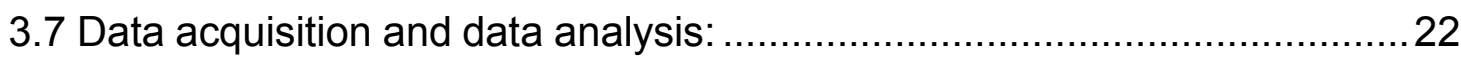

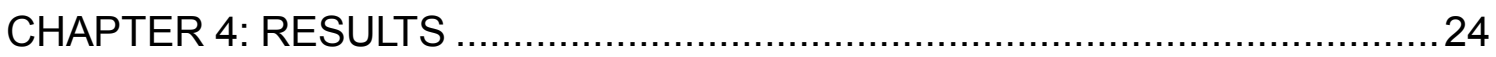

4.1 Determination of strength-duration curve parameters to set pulse width of

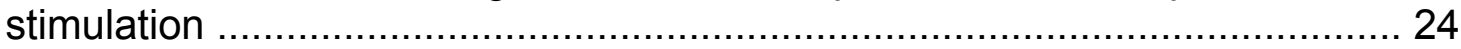

4.2 Adaptive pattern shaping capability of the closed-loop controller ............ 25

4.3 Ability of the closed-loop controller to counter muscle fatigue during combined muscle stimulation.................................................................... 28

4.4 Observation during sigh phenomena ................................................ 31

CHAPTER 5: DISCUSSION \& CONCLUSION .............................................. 35

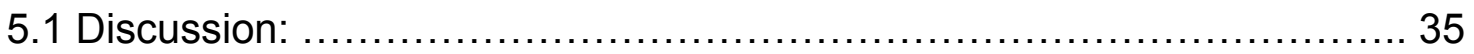

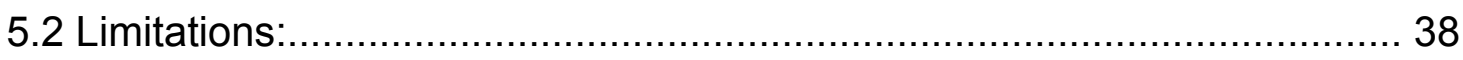

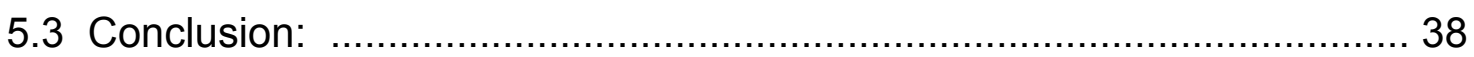

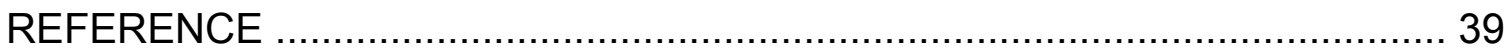




\section{ABBREVIATIONS AND ACRONYMS}

FES- Functional Electrical Stimulation

PG- Pattern Generator

PS- Pattern Shaper

SCl- Spinal Cord Injury

FI- Fatigue Index

iRMSE- Inspiratory Root Mean Square 


\section{INTRODUCTION}

Acute cervical cord injury, spinal artery infarction, brainstem disease, and stroke are usually associated with profound respiratory function compromise (Brian $\mathrm{J}$ Kelly and Luce 1991). Often, cervical spinal cord injury can lead to diaphragm paresis as injury at the cervical level can damage the phrenic motor pools which

innervate the primary inspiratory muscle, the diaphragm (Alilain et al. 2011). Moreover, quadriplegic and tetraplegic patients whose phrenic nerve nuclei are partially or fully intact and have functional diaphragm activities have degrees of ventilatory insufficiency because of lack of intercostal innervation (B. J. Kelly and Luce 1991; Ragnarsson 2007). Impairment in the autonomic control of respiration results in hypoventilation and may lead to death.

Mechanical ventilation has a viable role in acute respiratory assistance, yet it has associated drawbacks under long-term usages such as inspiratory muscle atrophy, impaired speech, constraints on mobility, and the need for tracheal intubation (Levine et al. 2008). Functional Electrical Stimulation (FES) of the phrenic nerve or directly the diaphragm muscle is an attractive alternative to the mechanical ventilator. The pacing creates negative intrathoracic pressure by diaphragmatic contraction, and hence, inhalation of air takes place (Ragnarsson 2007). With commercially-available systems, however, the current amplitude and frequency of the stimulation of the FES system need to be manually adjusted. In case of physiological demand, i.e. exercise or sighing, increase in tidal volume is 
required. In these events, manual adaptation of the specific stimulus parameters is a problem.

Siu et al. developed an adaptive closed-loop pacing system that stimulates the diaphragm muscle and can adapt to changes in musculoskeletal properties due to spinal cord injury (Siu et al. 2019). However, there are some limitations associated with this approach, such as diaphragm muscle fatigue induced by chronic stimulation, and inability to produce larger tidal volumes due to lack of support from additional respiratory muscles. To overcome these limitations, we adapted the approach for diaphragmatic stimulation used by Siu et al. to provide combined and synchronized stimulation of the external intercostal and diaphragm muscles and evaluated this enhanced system in in-vivo experiments on anesthetized rats.

\subsection{Specific Aim and Hypothesis:}

The main goal of this research was to extend the adaptive closed-loop pacing system developed by Siu et al (Siu et al. 2019) to include combined stimulation of the diaphragm and intercostal muscles. The specific aims were:

Aim 1: To investigate if combined and synchronized stimulation of the intercostal and diaphragm muscles could achieve a desired breath volume profile while reducing stimulation-induced diaphragmatic muscle fatigue. 
Aim 2: To automatically periodically elicit a sigh, an augmented breath, by pacing the diaphragm and the external intercostal muscles synchronously by increasing charge delivered to each muscle set during specific cycles.

\subsection{Significance:}

Although the previous study with the adaptive closed-loop pacing system could eliminate the need for constant adjustment of stimulation parameters, inconvenience of patients, and can attain eupneic breathing both in intact and spinal cord injured animals (Siu et al. 2019), it did not incorporate sighing, a vital breathing behavior. Moreover, functional electrical stimulation follows the reverse order of motor unit recruitment, increasing the propensity for rapid muscle fatigue (Stephens, Garnett, and Buller 1978). Therefore, the issue of muscle fatigue during chronic diaphragm pacing also needs to be addressed.

People breathing with a constant breath volume may experience atelectasis, the progressive collapse of alveoli, and reduced lung compliance (Vlemincx et al. 2009). Occasional deep breaths and sighs can restrain the lung from getting collapsed and restore lung compliance (Vlemincx et al. 2009). People dependent on mechanical ventilators also need the help of a ventilatory program that includes periodic sighs to combat alveolar collapse (Li et al. 2016). As the adaptive closedloop controller tries to achieve the desired breath volume with a constant period, atelectasis might occur during the stimulation-induced breathing. 


\subsection{Rationale:}

During diaphragm pacing, as the muscle contracts, it is accompanied by the inward movement of the rib cage, reducing the intrathoracic volume. This reduction in volume diminishes the efficiency of diaphragm stimulation to generate tidal breath volume. The goal of combined external intercostal and diaphragm muscle stimulation is to provide stabilization and expansion of the upper rib cage. The contribution of the external intercostal muscle contraction to attain tidal breathing during the adaptive stimulation of muscles may require a lower level of pacing induced diaphragmatic contraction to obtain the desired tidal volume and thereby reduce stimulation-induced fatigue in the diaphragm muscle. Furthermore, the synergized activation of the diaphragm and intercostal muscles could provide enough support to induce sighing behavior.

\subsection{Organization of the thesis:}

Chapter 1 represents a brief introduction, the significance of the investigation, rationale of the experiments and the specific aims. Chapter 2 presents a brief literature survey about physiological control of ventilation, motor unit recruitment during muscle pacing, and review of specific literature on respiratory muscle pacing. Chapter 3 describes surgical procedures for experiments, experimental protocols, data acquisition, and data analysis. Chapter 4 discusses the results or outcomes of the experiments, and chapter 5 contains the discussion and conclusion. 


\section{CHAPTER 2: BACKGROUND}

\subsection{Generation of respiratory rhythm:}

Spontaneous breathing patterns result from the integration of activity in the central nervous system, particularly in the brainstem, and feedback from central and peripheral chemoreceptors (Naik, Lynch, and Durbin 2015). Additionally, pulmonary stretch receptors, lungs-capillary receptors, and blood pressure affect ventilation (Naik, Lynch, and Durbin 2015). A group of neurons located in the rostral ventrolateral medulla called the pre-Bötzinger complex generates the respiratory rhythm (Lumb 2016). Under a proper level of oxygenation, the preBötzinger complex produces eupneic breathing rhythms (Wang-Rubin 2017). Eupnea or normal breathing is a low amplitude, fast frequency breathing rhythm which includes sighs which are bigger amplitude breaths interspersed at a lowfrequency. Previous research indicates that the Pre-Bötzinger complex produces a burst of activity during both eupneic and sigh breathing (Tryba et al. 2008). However, the comprehensive mechanism of sighing is still mostly unknown

The lungs are composed of hundreds of millions of alveoli that offer a large diffusive surface area across which gas exchange takes place during the process of breathing (Lumb 2016) . During normal breathing, alveoli continuously collapse during exhalation and expand during inhalation (Lumb 2016). However, to prevent atelectasis, which is the collapse of a large number of alveoli and ultimately the lung itself, eupneic breaths need to be interspersed with sighs. As a sigh is a large breath, it draws additional air to re-expand all the alveoli and prevent atelectasis. 
The occurrence of spontaneous sighing is modulated by feedback signals from pulmonary mechanoreceptors and peripheral chemoreceptors. The pulmonary mechanoreceptors sense atelectasis by changes in lung volume and transmural pressure and transmit afferent signals to the brainstem respiratory networks via the vagal nerve to trigger sighs (Naik, Lynch, and Durbin 2015). Besides, during alveolar collapse, as effectiveness of gas exchange decreases, hypoxic blood circulates in poorly ventilated pulmonary areas and triggers the chemoreceptors which too send additional drive to the brainstem respiratory networks ( $\mathrm{Li}$ and Yackle 2017).

The medullary respiratory center transmits descending impulses to the interneurons (DiMarco 2001). The final integration of the descending respiratory drive occurs at the motoneurons in the spinal cord (Butler 2007). The motoneurons at the cervical levels of the spinal cord innervate the primary inspiratory muscle, the diaphragm, via the phrenic nerve. Additionally, there are two types of inspiratory intercostal muscles: external intercostal and inter-cartilaginous portion of intercostal muscles, also known as parasternal intercostal muscles (De Troyer, Kirkwood, and Wilson 2005). Impulses from the respiratory center also synapse with the intercostal motoneurons located in the mid-cervical and thoracolumbar segments of the spinal cord, and these motoneurons innervate the inspiratory and expiratory intercostal muscles and abdominal muscles (DiMarco 2001). All the intercostal muscles are innervated by the corresponding intercostal nerve in any given interspace (De Troyer, Kirkwood, and Wilson 2005). 
The motoneuron is referred to as the final common output of the central nervous system to the muscle (Butler 2007). The neural drive to the motoneuron pool modulates the timing of firing and the firing rates of the motor units. Motor unit recruitment of the muscle follows the Henneman's size principle. The recruitment of the diaphragm muscle moves the abdominal wall outwards and expands the lower rib cage during inspiration. The changes in position result in an increase in intrathoracic volume and decrease in the intrathoracic pressure. Subsequently, air flows into the lungs and inflates the alveoli, where the exchange of gases takes place. In a non-disabled person, the diaphragm muscle contraction accounts for about $65 \%$ of the tidal volume production (Jarosz et al. 2012). The intercostal muscles are widely distributed throughout the rib cage. The intercostal muscles provide expansion and stabilization during the inspiratory phase. Though the diaphragm is the primary inspiratory muscle, contraction of the inspiratory intercostal muscles results in generation of about $35-40 \%$ of inspiratory volume capacity (DiMarco 2001).

Expiration is usually a passive process where inspiratory muscles relax. The pressure gradient between atmosphere and the lungs drives out the air from the lungs until it reaches equilibrium. Rectus abdominus, transversus abdominus, internal intercostal, pectoralis major are the expiration muscles (De Troyer, Kirkwood, and Wilson 2005). 


\subsection{Motor unit recruitment of respiratory muscles:}

The forces generated by the diaphragm muscle during ventilation are mostly dependent on the number and type of motor unit recruitment. According to the properties of the muscle fibers, the motor unit is commonly classified into three types: Type Slow (S), type Fatigue intermediate (Fint), and type Fast Fatigable (FF). The Type S motor unit has a slower contraction time and is more resistant to fatigue(Sieck and Ferreira 2013). Type Fint and FF have larger fibers and are more fatigable. Recruitment of motor units generally matches their fatigue properties (Mantilla and Sieck 2011). During eupnea, respiratory muscles usually recruit only

type $S$ fibers. But in case of other non-ventilatory behaviors i.e. coughing and sneezing, where more forces are needed, progressive recruitment to Fint and FF type motor units also takes place (Mantilla and Sieck 2011). In contrast, motor units follow the reverse order of recruitment process during electrical stimulation (Fang and Mortimer 1991).

\subsection{Techniques to restore impaired ventilation:}

Spinal cord injury or impairment in the neural control of breathing may result in paresis (reduced ability to activate motoneuron) or paralysis (inability to activate motoneuron) of respiratory muscles and can lead to respiratory insufficiency (Galeiras Vazquez et al. 2013). A patient with respiratory failure needs emergency support through mechanical ventilation. Though mechanical ventilation aids as a lifesaving tool in emergency cases, it is associated with some disadvantages under chronic usage such as respiratory muscle atrophy, need for placement of invasive tubing in the trachea, and associated impairment in mobility. Though development 
in improving mechanical ventilation is continuing, shortcomings persist. (Levine et al. 2008)

The most common and effective method for chronic restoration of inspiratory muscle function is diaphragm pacing (DP). Diaphragm pacing can be done by phrenic nerve stimulation, intramuscular stimulation or by spinal cord stimulation (DiMarco 2005). Compared to mechanical ventilation, phrenic nerve pacing systems offer more physical and psychological comfort, mobility, and social interactions (Ragnarsson 2007). Currently, three phrenic nerve pacing systems are available commercially: Avery Breathing pacemaker system by Avery Laboratories (Commack, New York, NY, USA) (Ragnarsson 2007), the Atrostim Phrenic Nerve Stimulator by Atrotech (Tampere, Finland), and the Medlmplant by Medlmplant Biotechnisches Labor (Vienna, Austria). All these systems have phrenic nerve stimulating electrodes implanted bilaterally in the cervical or thoracic regions. These systems also include a radiofrequency stimulator which receives a radio frequency signal from an externally located circuit. The stimulation of the phrenic nerve results in diaphragmatic contraction and outward movement of the abdominal wall, which creates negative intrathoracic pressure. The change in pressure gradient draws air from the atmosphere to the lungs. As the stimulation stops, the diaphragm relaxes and ascends, which results in exhalation. The amplitude and frequency of the stimulus need to be adjusted manually. The Avery system uses monopolar, or bipolar nerve cuff electrodes and the Atrostim system uses a four-pole sequential electrode. The four electrodes contacts by evenly spaced on the phrenic nerve and sequentially activate different parts of the nerve 
and hence diaphragm muscle. The sequential stimulation of the nerve aims to reduce the diaphragm muscle fatigue. The Medlmplant uses 'Carousel' stimulation, where four electrodes are sutured to the epineurium of each phrenic nerve. Those four electrodes also sequentially stimulate the phrenic nerve and reduce stimulation induced muscle fatigue (Ragnarsson 2007).

There are some risks associated with phrenic nerve pacing, which includes surgical risks and possible damage to the phrenic nerve. In contrast, the intramuscular diaphragm stimulation approach offers less surgical complication than phrenic nerve pacing as it does not involve thoracotomy and placement of an electrode on the phrenic nerve, which removes the risk of possible phrenic nerve damage. For intramuscular diaphragm stimulation, the diaphragm can be accessed laparoscopically, and it requires the placement of an electrode near the motor point of the phrenic nerve to activate the diaphragm muscle. However, as large fatigable fibers in the muscle have lower stimulation threshold, electrical stimulation of the muscle leads to rapid fatigability(Fang and Mortimer 1991).

Many researchers conducted animal studies to get maximal respiratory responses by stimulating multiple respiratory muscles (DiMarco 2001, 2005; Dimarco, Budzinska, and Supinski 1989; Walter et al. 2011, 2017). DiMarco et al. showed that activating intercostal muscles by spinal cord stimulation in $\mathrm{T} 2$ region could generate large inspiratory volume (Dimarco, Budzinska, and Supinski 1989). Various studies with animals recommend that intercostal muscle stimulation can be a useful technique for inducing inspiration (DiMarco 2001). Moreover, a 
previous chronic study in a canine model where the inspiratory muscles (i.e. diaphragm muscle, abdominal muscle and upper thorax muscles) were stimulated alone and then in combinations (diaphragm \& abdomen, diaphragm \& thorax and all three muscles together) via permaloc electrodes showed that combined muscle stimulation resulted in larger inspiratory breath volume than the volume induced by the individual muscle alone (Walter et al. 2011). However, activation of intercostal muscles is more challenging than of the diaphragm as there are twelve pairs of intercostal muscles on each side of the thorax. Another optimization test was conducted by Walter et al. in a canine model to determine stimulation of which intercostal muscles between the 1st to 4 th intercostal spaces yielded maximum inspiration (Walter et al. 2017). The result of the test suggested that the surface electrode located at the 2nd intercostal space showed the most substantial inspiratory volume.

Though sighs can reset regular breathing and can improve compliance and gas exchange in alveoli (Naik, Lynch, and Durbin 2015), very few studies have been done to incorporate sighing in functional electrical stimulation or mechanical ventilation. A study of mechanical ventilation incorporated with periodic sighs showed that in acute respiratory failure sighs decrease lung strain and ventilation heterogeneity (Mauri et al. 2015). Studies also showed that optimal lung inflation and improved gas exchange could be provided with cyclical sighs during mechanical ventilation in acute respiratory syndrome patients (Patroniti M.D. et al. 2002; Pelosi et al. 2003). 


\subsection{An Adaptive ventilation control system:}

Though there are many systems available which can provide support to create tidal breath volume, all the systems are open-loop stimulation systems. But ventilation itself is variable and not only depends on respiratory factors but also some other non-respiratory factors like posture, locomotion, changes in metabolic demand and voluntary activities (Lumb 2016). So, the open-loop systems require frequent manual input of the stimulation parameters. To address this limitation, Siu et al. developed an adaptive closed-loop stimulation system which can adapt the stimulation to the diaphragm in response to changes in musculoskeletal properties of muscle following a spinal cord injury (Siu et al. 2019). In animal studies, intramuscular diaphragm stimulation with the closed-loop adaptive system was able to restore a desired breath-by-breath tidal volume profile in spinal cord injured (C2 hemisected) animals. However, in this study only the diaphragm muscle was stimulated and the ability to generate large breath volume like sighs was not explored. For our current investigation, we adapted the closed-loop controller for intramuscular diaphragm and intercostal stimulation to reduce muscle fatigue during chronic muscle stimulation and to induce sighing. 


\section{CHAPTER 3: METHODS}

\subsection{Surgical procedures:}

All procedures followed approved guidelines established by the Institutional Animal Care and Use Committee of Florida International University. Rats were housed in the university animal care facility with a 12-hour light/dark cycle, with ad libitum access to food and water.

Experiments were conducted on eight anesthetized, spontaneously breathing, adult male Sprague Dawley rats weighing $400 \pm 80 \mathrm{~g}$. Rats were maintained under anesthesia with subcutaneous urethane $(50 \mathrm{mg} / \mathrm{kg}$ ) injection followed by supplementary isoflurane $(0.5-3 \%)$ inhalation. Toe pinch reflex and monitoring of respiratory rate were used as an indicator of the proper level of anesthesia. The body temperature of the animal was maintained around $37^{\circ} \mathrm{C}$ with the help of a closed-loop thermal pad and a rectal thermometer. For monitoring heart rate of the animal, 30G stainless steel needle electrodes were implanted subcutaneously on chest 1-1.5 inch apart and the electrocardiogram was recorded. A chest mounted respiratory belt was utilized to monitor the breathing pattern and breathing rate of the animal. To avoid dehydration, a lactated ringer solution was injected subcutaneously every 2-3 hours. When the animal was fully anesthetized, the surgical procedures were commenced.

The surgery was done in the supine position. A tracheostomy was performed to insert a short custom tracheal tube made of flexible, hollow plastic tube. A small pneumotachometer (PTM type HSE-73-0980) was directly connected to the 
tracheal tube to measure airflow. This flow was integrated (PI-1000, CWE Inc. Ardmore, PA, time constant $=0.2 \mathrm{~s}$ ) to obtain the breath volume of the animal on a breath-by-breath basis. A capnograph (CapStar-100, CWE Inc., Ardmore, PA) measure end-tidal $\mathrm{CO}_{2}\left(\mathrm{etCO}_{2}\right)$ throughout the experiment. To implant pacing electrodes in the diaphragm muscle, a $2-2.5 \mathrm{~cm}$ incision was made rostral to caudal from the xiphoid process. After having access to the peritoneal cavity, a hand-operated stimulator (DigiStim 3 plus, Neuro Technologies), providing a single monophasic pulse at $1 \mathrm{~Hz}$ was used to stimulate the diaphragm muscle. The stimulation was used to map the location of motor points in the diaphragm, by visualizing muscle twitch of both the hemi-diaphragms. One electrode in each hemi-diaphragm was implanted near the point where the strongest contraction was observed. The electrodes used were custom-made; a $20-25 \mathrm{~cm}$ long stainless steel wire (Diameter of the wire is 0.002 " (bare), 0.0045 " (coated)), with about $2 \mathrm{~mm}$ exposed length at the end where the wire was inserted into muscles and about 5$8 \mathrm{~cm}$ exposed length where the wire was connected to a suture needle $(10 \mathrm{~mm}$, 3/8th circle, cutting, Havel's Inc., Cincinnati, OH).

An incision was made along the $2^{\text {nd }}$ to $3^{\text {rd }}$ intercostal space for implanting an electrode in the $2^{\text {nd }}$ intercostal space. Muscle layers were removed by blunt dissection. Having access to the external intercostal muscle, again, the handoperated stimulator was used to identify a motor point. The same procedure was followed to implant electrodes in the left and right external intercostal muscles and determine the twitch threshold. 


\subsection{Determining pulse width duration of stimulation for the inspiratory muscle pacing}

As the threshold for activation of muscle fiber depends not only on the stimulation strength but also the duration of stimulus, to set the stimulation pulse width, we needed to illustrate strength duration curve for the diaphragm and external intercostal muscles. The optimum pulse width duration for activation of the inspiratory muscles was determined by rheobase and chronaxie from strengthduration curve. To find out the strength-duration curve, we stimulated the left and right side of diaphragm muscle and external intercostal muscle separately at 75 Hz. At a fixed stimulation pulse width, we modulated the stimulation strength. The minimum stimulation amplitude where muscle twitch was observed, was the twitch threshold for the muscle fiber for that given pulse width. Same procedure for determining twitch threshold was repeated for pulse width in the range 10-500 $\mu \mathrm{s}$. Curve-fitting procedure for the strength-duration data provided the strengthduration curve for both sides of diaphragm and external intercostal muscle for $n=2$ animals. The minimum current amplitude required to activate the muscle fiber was the rheobase and the time associated with twice the rheobase is chronaxie. Chronaxie provided us the optimum pulse duration for the muscle fiber activation.

\subsection{Diaphragm, external intercostal muscle pacing}

The twitch threshold current (the minimum current required to elicit visible muscle contraction) was determined for each electrode by visualizing muscle contractions as stimulation pulses were sent using a programmable constant-current stimulator 
(FNS-16, CWE Inc., Ardmore, PA) delivering cathodic first, biphasic current pulses of $80 \mu \mathrm{s} /$ phase at $75 \mathrm{~Hz}$, varying the current amplitude. The twitch threshold confirmed the proper placement of the electrode within the muscle. The twitch threshold of the muscle informed us the relative excitability of the muscle and directed us to set the maximum current amplitude of the diaphragm muscle and the external intercostal muscle. The maximum current amplitude was set as 1.5 2 $\times$ twitch threshold of the muscle. The maximum current amplitude was set 2-4 mA. We kept maximum current amplitude of the stimulation same for both side of the diaphragm muscle and external intercostal muscle.

Adaptive pacing was performed using the same programmable FNS-16 stimulator using cathodic first, biphasic current pulses of $80 \mu \mathrm{s} /$ phase pulse width with $40 \mu \mathrm{s}$ interpulse interval at $75 \mathrm{~Hz}$. The current amplitude was modulated by the adaptive pattern shaper (PS) controller every $40 \mathrm{~ms}$. The output of the PS unit is in the range of 0 to 1 , and a multiplier with maximum allowed amplitude multiplies the PS output and sends a command to the stimulator (see figure 1). At the initiation of a pacing trial the current amplitude starts at zero, and then the controller starts to adapt the stimulation amplitude.

\subsection{Experimental protocol}

The adaptive controller modulates the stimulation parameters to match the desired breath volume on a breath-by-breath basis with a fixed breath duration (cycle period). Each trial consisted of at least one minute of spontaneous breathing, followed by at least five minutes and at best fifteen minutes of stimulation. For each 
trial, the desired breath volume profile and cycle period during pacing was obtained by averaging the pre-stimulation recorded breathing cycles except for nonbreathing behavior cycles, i.e., sighs. As intact rats have an intrinsic breathing pattern, successful entrainment of the stimulation assisted breath with the intrinsic breath requires the stimulation assisted breath to have a larger tidal volume. Presumably, the pulmonary stretch receptor feedback to the central respiratory pattern generator during this larger amplitude breath helps entrain the two. So, to get entrainment, the desired breath volume needed was set at an ad-hoc $120 \%$ of the baseline volume (Siu. et al. 2019). Experimental trials were spaced 20-30 minutes apart in order to allow the inspiratory muscles to recover from stimulationinduced fatigue. The baseline volume recorded prior to the stimulation was directing us to change in tidal volumes. Two consecutive recorded baseline volume was not much different, confirming sufficient allowed rest period between trials. The order of the diaphragm only vs. combined stimulation trials was changed randomly.

To induce sighs, the adaptive learning in the controller was paused during the sigh cycle and the following cycle. The controller stored the PS outputs (time shifted weighted summation of neuronal output) of each update for the previous breath cycle and sends out double of the magnitude of PS output to the maximum amplitude multiplier in the controller. The double of the amplitude of stimulation on each instant assists the inspiratory muscles to contract to an additional degree. The frequency of the sigh was set at every 30 breathing cycles. 


\subsection{The PG/PS controller}

The controller was originally developed by Abbas and Chizeck (Abbas and Chizeck 1995), used in an incomplete spinal cord-injury (iSCl) rodent model for cyclic limb movement (Fairchild et al. 2010) and in an iSCl rodent model for functional stimulation of the diaphragm muscle to attain a desired breath volume pattern (Siu et al. 2019).

The controller is a neural network system that can provide automated customization of the stimulation parameters to drive the desired action. It has two components: a pattern generator (PG) and a pattern shaper (PS). The PG has a pattern generating capability of neurophysiological models and outputs a periodic signal. In this study, a frequency oscillator was used to produce a fixed respiratory frequency where the period did not change from breath-to-breath (Siu et al. 2019). The PS is a single-layer artificial neural network that takes its input from the PG and provides the parameters for stimulation of the muscles. The PS unit adapts the stimulation parameters (current pulse amplitude) to attain a desired breath volume trajectory. The adaptation occurs based on the error between the measured breath volume and the desired breath volume every 40 msec (time step) within a breath. The output of the PS unit at each time step is the weighted summation of neuron outputs. In this study, the number of neurons was set to 25 . As the stimulator operates at $75 \mathrm{~Hz}$, each update from the controller results in three pulses of the same amplitude before a change in pulse amplitude. Each neuron

output reaches its peak amplitude every $40 \mathrm{~ms}$, and it overlaps with a specified 
number of neighboring neurons in the time domain. The output of the PS at each time step can be defined as,

$$
\mathrm{Z}(\mathrm{t})=\sum_{j=1}^{n_{a}} w_{j}(t) y_{j}(t)
$$

Where, $y_{j}(t)$ is the output of the neuron $j$ at time $t$ and $w_{j}(t)$ is the weight for the specific neuron. The output $\mathrm{Z}(\mathrm{t})$ is in the range of values between 0 and 1 and then scaled by the maximum current amplitude defined for the muscle stimulation.

The weighting factor is modified based on the instantaneous error between the target (desired) breath volume and the measured volume. The change in weights for neuron $j$ can be defined as

$$
\Delta w_{j}(t)=\eta e(t) \sum_{i=1}^{n_{p}} \frac{1}{n_{p}} y_{j}(t-i T)
$$

Where $\eta$ is the learning rate, in this study $\eta=0.001, e(t)$ is the instantaneous error at time $t, T$ is the update period $=40 \mathrm{msec} n_{p}$, is the number of past activations over which the error was averaged $=6$. For our experiments, one PS unit was used to stimulate both sets of muscles. 


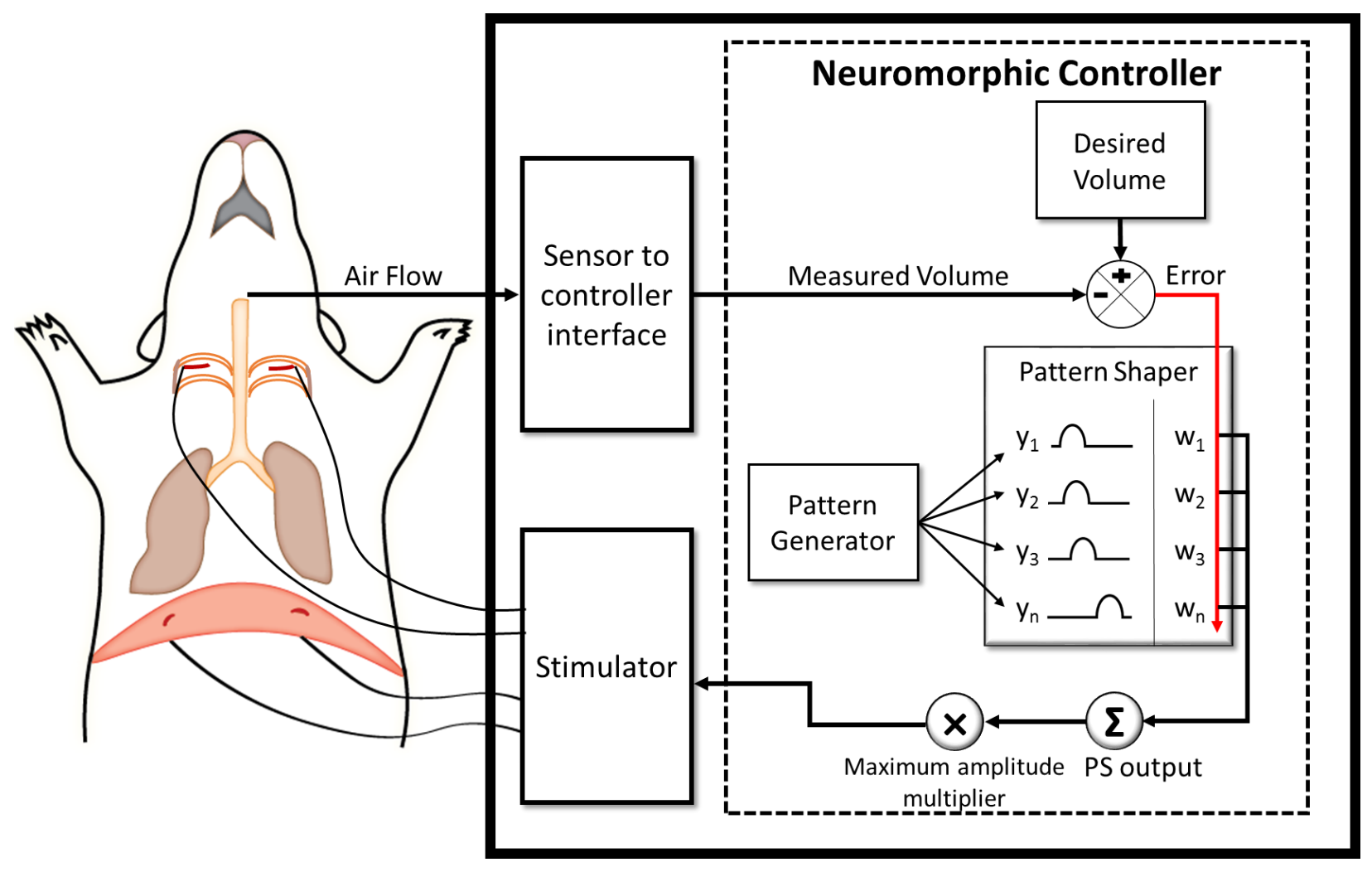

Figure 1: The adaptive neuromorphic controller architecture (Modified version of a figure from (Siu et al. 2019) ).

Bilaterally electrodes were implanted in the diaphragm and external intercostal muscles. Pattern generator is a fixed oscillator to generate fixed period respiratory cycles. Pattern shaper is the artificial neural network, adapts based on the instantaneous error between desired and measured volume. The summation of the time shifted neuronal output gets multiplied by the maximum amplitude of stimulation and sends information to the stimulator for stimulating the inspiratory muscles.

\subsection{Performance measures}

For evaluating the tracking performance of the controller, the error was calculated based on the desired breath volume and the measured breath volume at a given instant. In order to quantify the error, percent inspiratory root-mean-squared error was calculated for this given equation 


$$
i R M S E=\frac{100}{\max \left(V_{D}\right)} \sqrt{\frac{1}{q} \sum_{i_{I N S P}}^{q} e_{i^{2}}}
$$

Where $\max \left(V_{D}\right)$ is the peak value for the desired volume, iINSP represents the time step at the beginning of inspiration, $q$ is the number of time steps in the inspiratory phase of the desired breath volume profile, and $e_{i}$ the instantaneous error at time step $i$.

To quantify charge delivered per cycle during each cycle of the trial, we calculated charge delivered per cycle as the total summation of the product of the width and amplitude of each pulse sent during one cycle. A fatigue index of each trial was calculated as the difference in average initial charge delivered per cycle and final charge delivered per cycle, normalized by the initial charge per cycle. The initial and final cycle set consisted of about 50 cycles. We considered the initial charge per cycles when the measured breath volume and desired breath volume started to match. The initial charge per cycle set and the final charge per cycle set was about 400 cycles apart and set was considered such a way that there is no to negligible number of loss of synchrony cycles. For animal 1 and animal 2, the recording time was short, fatigue index was calculated for all the animals except 1,2. We calculated fatigue index for combined muscle stimulation trials and only diaphragm muscle stimulation trials. Fatigue index is representing relative increase in charge delivered to the muscles during one trial. As the muscle gets fatigued by the stimulation provided during the trial, to maintain same functional level, charge delivered to the muscle get increased. So, the relative increase in charge delivery 
or fatigue index was used as a measure of muscle fatigability during the stimulation of one trial.

Since tidal volume depends on the weight of the animal and we got the desired volume from the baseline recording before any stimulation, the desired tidal volume varied along all rats and sometimes for different trials for the same rat. We normalized the sigh tidal volume data with the desired tidal volume and denoted this as "tidal volume factor".

\subsection{Data acquisition and data analysis:}

Each recording trial was from 5 to 15 minutes long, consisting of at least 60 seconds of intrinsic breathing, followed by stimulation augmented breathing. At least 20 to 30 minutes resting period was allowed between trials. Recordings were obtained during only diaphragm muscle stimulation, during combined muscle stimulation, and separate trials were taken for inducing sigh with only diaphragm muscle stimulation and combined muscle stimulation. Data recording included EKG, breath volume, charge delivery, end-tidal $\mathrm{CO}_{2}\left(\mathrm{etCO}_{2}\right)$, and PS output (Normalized simulation output). All the data from the PG/PS controller were collected at $25 \mathrm{~Hz}$, and other recorded data were collected at $10 \mathrm{kHz}$, which later on was down-sampled to $25 \mathrm{~Hz}$. Statistical analysis was conducted by paired sample t-test in SPSS. One-way paired sample t-test analysis was conducted on fatigue index data with $\alpha=0.05$ significance level to test if charge delivered per cycle was significantly lower in combined muscle stimulation. The tidal volume 
factor of sighs was averaged for combined muscle stimulation and only diaphragm muscle stimulation. One-way paired sample t-test was performed on the averaged tidal volume factor to test if tidal volume generated for the sigh cycles were significantly greater in combined muscle stimulation than only diaphragm muscle stimulation. 


\section{CHAPTER 4: RESULTS}

\subsection{Determination of strength-duration curve parameters to set pulse width}

\section{of stimulation}

For $n=2$ animals, strength duration curve was illustrated to determine the optimum pulse duration. Figure 2B shows that during longer pulse widths, the strengthduration curve approaches linear behavior, at this point even though pulse width was increased, stimulation amplitude did not decrease much. The minimum current amplitude required to activate muscle fibers provided us the rheobase. And the time associated with twice as the rheobase is chronaxie (pointed by dashed line in figure 2). For both inspiratory muscles, chronaxie value was about $80 \mu \mathrm{s}$. Thus, the stimulus pulse width for optimum response by eliciting muscle was determined.
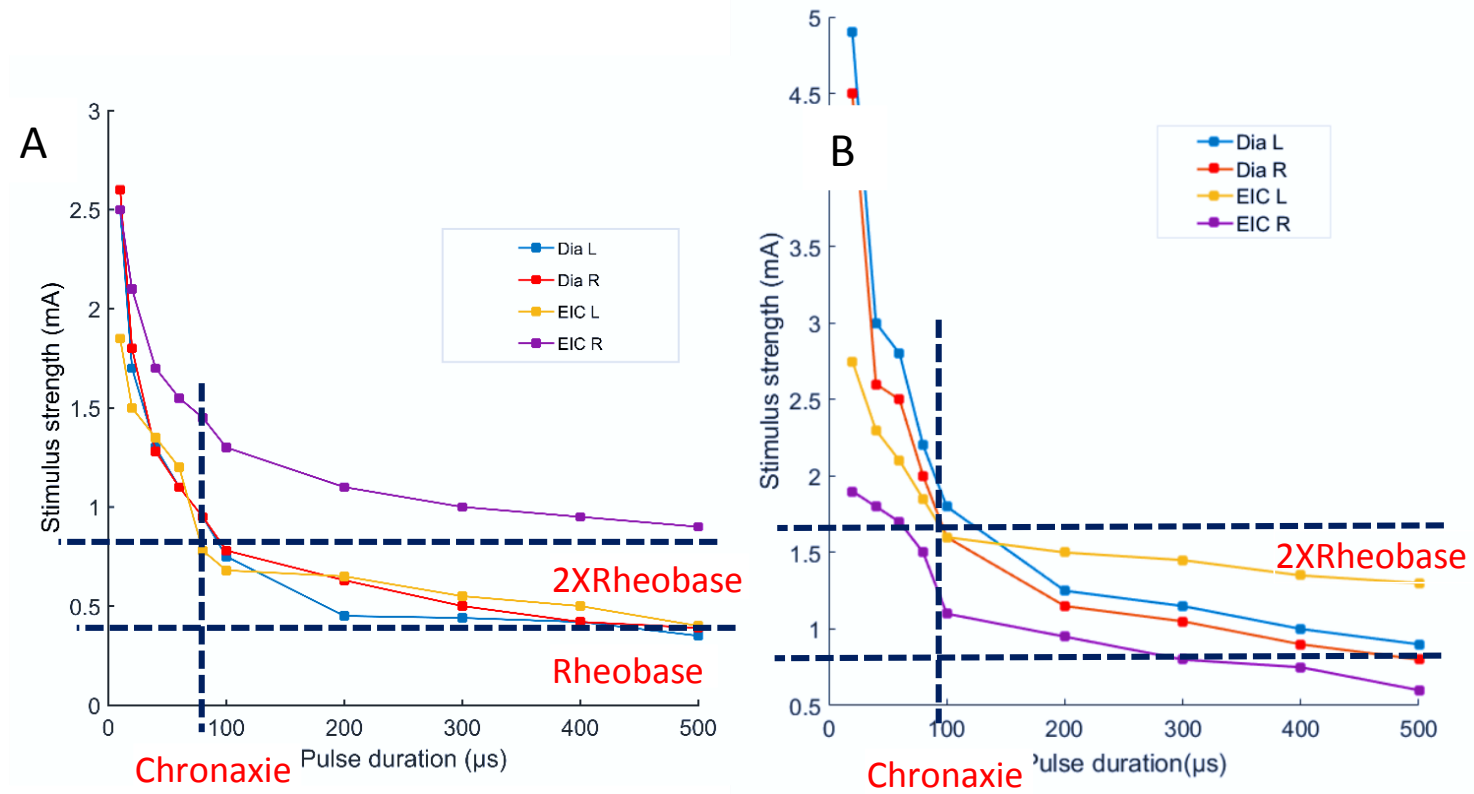
Figure 2: Strength-duration curve for left, right diaphragm muscle and external intercostal muscle.

$A, B$ are strength duration curves for 2 animals. Figure $A$ and $B$ are showing rheobase and chronaxie value (by a blue dashed line) for left external intercostal muscle and figure $B$ is showing rheobase and chronaxie for left diaphragm muscle.

\subsection{Adaptive pattern shaping capability of the closed-loop controller}

We performed diaphragm stimulation or combined muscle pacing of the diaphragm and external intercostal muscles to compare and assess the effect of the synergized activation of respiratory muscles during combined muscle stimulation on the breath volume profile. Figure 1 shows the outcome during stimulation of respiratory muscles with the adaptive closed-loop controller. Figure $3 \mathrm{~A}, \mathrm{C}$ show two representative graphs at the time when the controller is turned on, for the diaphragm muscle stimulation and combined muscle stimulation respectively. The stimulation output (Stim.(mA)) initially starts at zero when the controller is turned on. The controller adapts and tries to match the stimulation assisted breath volume profile with the desired breath volume profile by increasing the charge delivery. Figure $3 \mathrm{~B}$ and $\mathrm{D}$ show continuation of figure $\mathrm{A}$ and $\mathrm{C}$ respectively for the last 10 seconds of the trials for diaphragm muscle stimulation and combined muscle stimulation. Here, the assisted breath volume profile matches the desired breath volume profile. Figure 3E shows an example of the pattern shaping capability of the controller for the first 100 s of a 15 minutes long trial for combined muscle stimulation. The vertical light blue dashed line at the beginning of the trial shows the time when the controller started adapting. The inspiratory root mean square (iRMSE) value increases to account for the difference in volume between the 
stimulation assisted breath and the desired breath. Once the stimulation assisted breath volume profile starts to match the desired breathing pattern, iRMSE as well as the stimulation output start decreasing. For our investigations, when the iRMSE value decreased to $10 \%$ or less and remained there for at least 20 pacing cycles, the controller was considered to have adapted. This is indicated in Figure 1E by the second vertical dark blue dashed line. 


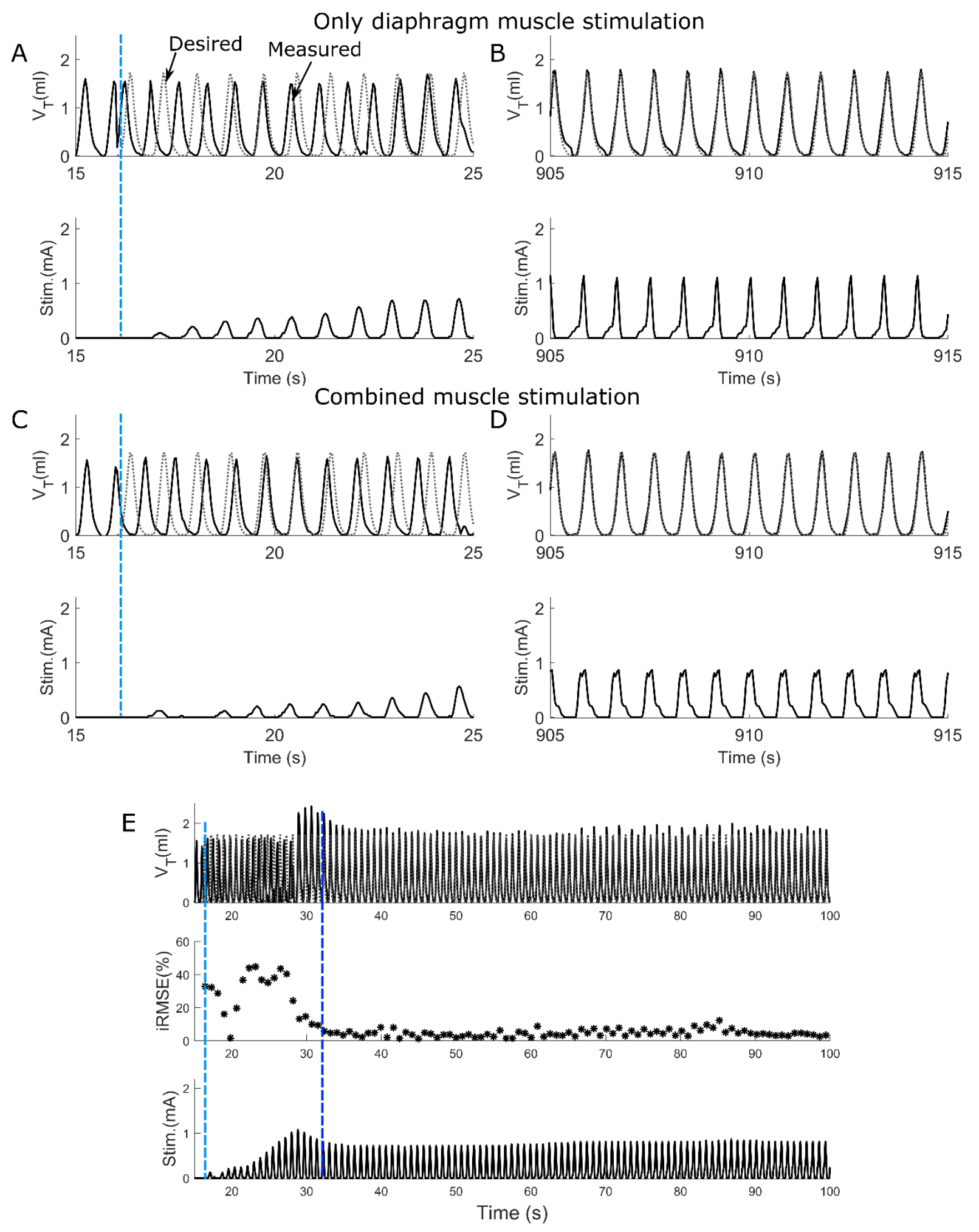


Figure 3: Ability of adaptive PS controller to attain the desired breath volume in intact animal during diaphragm muscle stimulation or combined muscle stimulation.

(A, C) Response to adaptive closed-loop control of diaphragm muscle stimulation or combined muscle stimulation respectively in a rat (rat 3). Stimulation output progressively increases to counter the mismatch between the measured stimulation assisted breath volume trajectories (solid line) and the desired breath volume trajectory (dotted line). The vertical light blue dashed line shows when the controller was turned on. (B, D) Continuation of $A$ and $C$ respectively showing last $10 \mathrm{~s}$ of the trial for diaphragm muscle stimulation and combined muscle stimulation. In both cases the stimulation assisted breath volume profile overlaps the desired breath volume profile. E The adaptive closedloop controller implementation for the combined muscle stimulation (same animal, same trial as C, D) for first $100 \mathrm{~s}$. At the beginning iRMSE was high; to match the elicited instantaneous volume with the desired instantaneous volume, stimulation output adapted and initially increased cycle by cycle. The second vertical dark blue dashed line shows where the stimulation assisted breath volume started to match the desired volume. iRMSE value decreased to $<10 \%$ and stimulation output was stable for the rest of the time.

\subsection{Ability of the closed-loop controller to counter muscle fatigue during}

\section{combined muscle stimulation}

Figure $4 \mathrm{~A}$ shows a representative figure of \%iRMSE for an experiment (rat 6) during either diaphragm muscle stimulation alone (in blue) or combined muscle stimulation (in red). It shows that initially \%iRMSE was high but once the controller adapted, \%iRMSE value decreased. Note that the overall iRMSE was higher for diaphragm muscle stimulation alone. Additionally, the \%iRMSE cycled between a high and low value from one breath to the next. Figure 4B shows charge per cycle delivered $(\mathrm{mC})$ to the diaphragm muscle during diaphragm muscle stimulation alone (in blue) or combined muscle stimulation (in red). During the later cycles of the trial (cycles 300-400), charge per cycle delivered increased, which suggests that to attain the desired volume, more charge needed to be delivered. This 
increase in charge delivered per cycle to attain the desired volume indicates the diaphragm muscle's fatigue susceptibility over the time of the trial.

Figure 4C shows the fatigue index (FI) for six animals (\#3-\#8). The diagonal dark blue line through the origin $(0,0)$ represents the line where the fatigue index is the same for the diaphragm stimulation alone $\left(\mathrm{Fl}_{\mathrm{dia}}\right)$ or combined muscle stimulation of the diaphragm and external intercostal (F/dia+ic). A positive fatigue index indicates that greater charge delivery was required after the controller had adapted while a negative fatigue index indicates that a lower charge delivery was required after the controller had adapted. The blue shaded area represents the region where the fatigue index was higher when only the diaphragm muscle was stimulated. Also, data close to the origin $(0,0)$ suggests that there was low amount of diaphragm muscle fatigue during stimulation. Figure $4 \mathrm{C}$ illustrates that most of the data was in the blue shaded area which suggests that diaphragm muscle was more fatigued during only diaphragm muscle stimulation than under combined muscle stimulation. As the number of sample data is very small $(n=6)$, we performed a normality test for the data for fatigue index for both combined muscle stimulation and only diaphragm muscle stimulation. Both the datasets showed normal distribution and hence we performed a one tailed paired sample t-test (2 dependent samples) in SPSS. The statistic test showed that FI values of the diaphragm muscle were significantly lower under combined muscle stimulation $(p<0.05)$. 

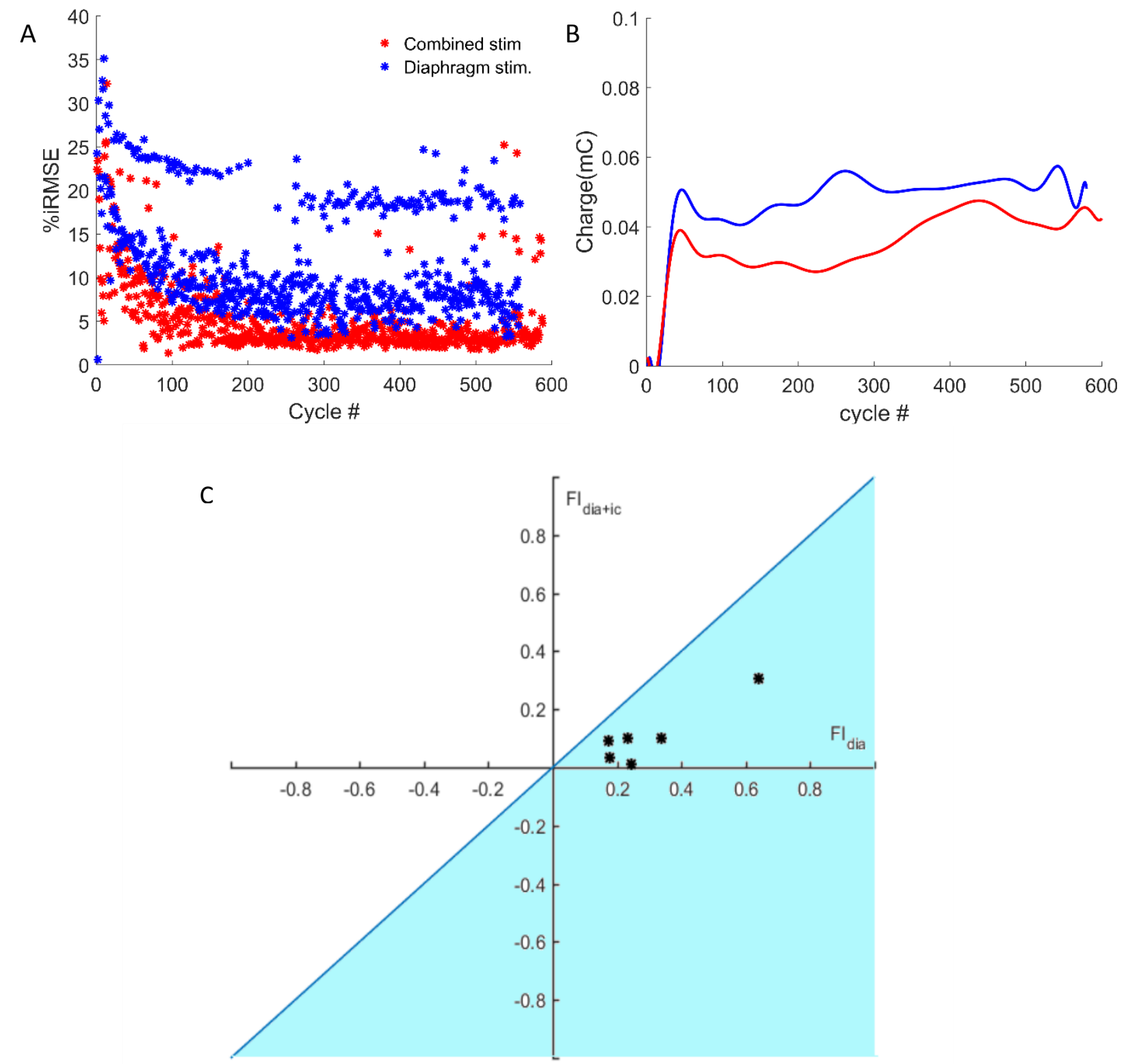

Figure 4: \%iRMSE and charge delivered per cycle to the diaphragm muscle and fatigue index representation during combined muscle pacing and diaphragm pacing.

A An example of \%iRMSE for diaphragm muscle stimulation alone (in blue) or combined muscle stimulation (in red), in an experiment (rat 6). At the beginning of a trial \%iRMSE was high, but once the controller adapted and the desired volume trajectory was obtained, the \%iRMSE value decreased. B An example of charge delivered per cycle to the diaphragm muscle for only diaphragm muscle stimulation (in blue) and combined muscle 
stimulation (in red) in the same trials as in A. When there was mismatch between the measured volume and the desired volume profiles, \%iRMSE value was high and to attain the desired volume profile increased charge was delivered to the muscles. $\mathbf{C}$ Fatigue index for only diaphragm muscle stimulation $\left(\mathrm{FI}_{\mathrm{dia}}\right)$ and combined muscle stimulation $\left(\mathrm{FI}_{\mathrm{dia}+\mathrm{ic}}\right)$. The blue line going through origin presents ratio of $\mathrm{Fl}=1$, any point below the line (the light blue area) suggests greater fatigue susceptibility of the diaphragm muscle during only diaphragm muscle stimulation.

\subsection{Observation during sigh phenomena}

We performed diaphragm muscle stimulation and the combined diaphragm, intercostal muscle stimulation synchronously to generate sigh-like behavior. Figure $5 \mathrm{~A}$ shows the volume profile during an intrinsic sigh recorded during an experiment overlapped with the volume profile of a previous regular breathing cycle. The illustration shows the peak volume of sigh is larger than that for the regular breath cycle. This information about sighs was utilized to generate a desired sigh during pacing. Since, we are using a constant frequency oscillator as PG, which gives constant period for all the desired cycles, sighing cycles were also of the same period as the desired cycle. It was then determined if the combined muscle stimulation could yield a larger tidal breath volume. Figure $5 \mathrm{~B}$ and $5 \mathrm{C}$ show the measured breath volume overlapping a desired breath volume profile for non-sigh cycles. During diaphragm stimulation alone or combined stimulation induced sigh, a larger measured volume was obtained mimicking the larger breath volume sigh.

A sigh functions as a re-setter for the intrinsic breathing. The intrinsic central pattern generator gets feedback during a sigh, since the large breath during a sigh activates pulmonary stretch receptors. Even for elicited sighs, if there had been a loss of synchrony between the intrinsic breathing and stimulation assisted 
breathing before a sigh, the sigh helped in resting the intrinsic breathing pattern and align the desired and measured volume profiles. In our experiments, in 12 sighs during different trials in different animals, we observed 11 sigh cycles (91\%) reset the alignment. If there were at least 3 breath cycles where the measured and desired breath initiation was out of phase, we considered that as loss of synchrony prior to a sigh cycle. Figure 5D shows an example of a sigh aiding in the alignment and synchronization of the desired and measured volume profiles. Prior to the sigh the measured volume profile is unsynchronized with the desired volume profile. Even though, high tidal volume could not be achieved by the stimulation provided for sighs, after the sigh induction the measured volume profile synchronized in time and matched the desired shape of the desired volume trajectory.

However, a large breath could not always reset and retrieve synchrony between the desired breathing profile and the measured breathing profile. Figure $5 \mathrm{E}$ shows an example where the measured stimulation assisted breathing got out of synchrony after a sigh cycle. A living being with breathing capacity may experience post sigh apnea, where right after a large sigh breath, breathing slows and exhibits spontaneous pauses to allow return of stable regular breathing (Yamauchi et al. 2008). In our trials for inducing sighs, adaptation of the controller was turned off during the sigh cycle and the following cycle, after which it resumed. Figure 5E shows that in this trial, rate of the intrinsic breathing pattern slowed down right after the sigh and the stimulation was unable to induce shortening of the intrinsic breath duration. Consequently, there was a loss of synchrony and lack of volume profile matching between the measured and desired volume trajectories. 
Figure 4F shows a graph for the normalized average tidal volume (measured sigh tidal volume/desired non-sigh tidal volume) obtained during diaphragm muscle stimulation (in blue) and combined muscle stimulation (in red) for seven rats. The table below shows the average tidal volume factor induced during combined muscle stimulation and only diaphragm muscle stimulation in 7 animals.

Table 1: Average tidal volume factor for combined muscle stimulation and only diaphragm muscle stimulation

$\begin{array}{cccc}\text { Rat \# } & \begin{array}{c}\text { Sample size, } \\ \mathrm{N}\end{array} & \begin{array}{c}\text { Average tidal } \\ \text { volume factor in } \\ \text { combined muscle } \\ \text { stimulation } \\ \text { (mean } \pm \text { S.D.) } \\ 1.60 \pm 0.09\end{array} & \begin{array}{c}\text { Average tidal } \\ \text { volume factor in } \\ \text { only diaphragm } \\ \text { muscle stimulation } \\ \text { (mean } \pm \text { S.D.) }\end{array} \\ 1 & 11 & 1.43 \pm 0.11 \\ 2 & 11 & 1.40 \pm 0.11 & 1.24 \pm 0.23 \\ 3 & 35 & 1.23 \pm 0.04 & 1.18 \pm 0.10 \\ 4 & 33 & 1.42 \pm 0.20 & 1.29 \pm 0.09 \\ 5 & 29 & 1.58 \pm 0.18 & 1.59 \pm 0.10 \\ 6 & 36 & 1.09 \pm 0.23 & 1.07 \pm 0.10 \\ 8 & 23 & & \end{array}$

In most cases, average tidal volume factors were larger in combined muscle stimulation than only diaphragm muscle stimulation. One-tailed paired sample ttest had been performed on the averaged tidal volume factors in both combined muscle stimulation and only diaphragm muscle stimulation and the t-test indicated that the averaged tidal volume factors in combined muscle stimulation was 
significantly higher than only diaphragm muscle stimulated tidal volume factors $(p=0.035<0.05)$.

A
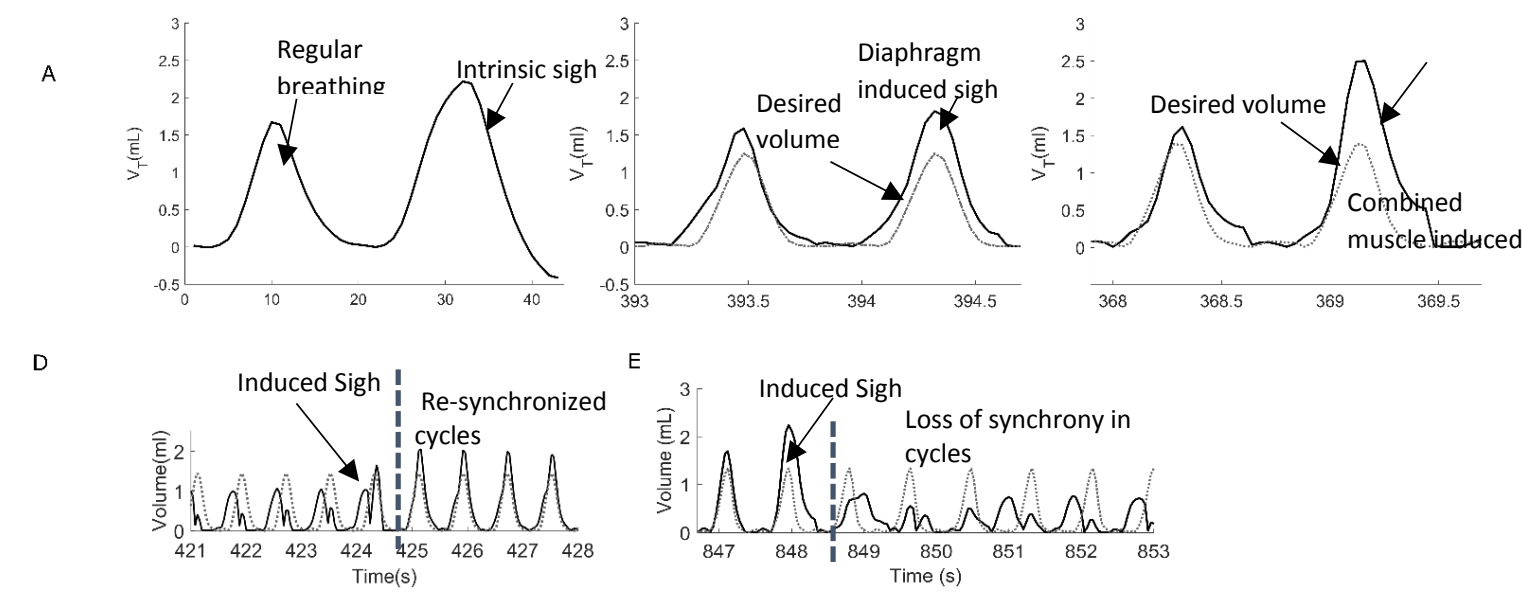

E
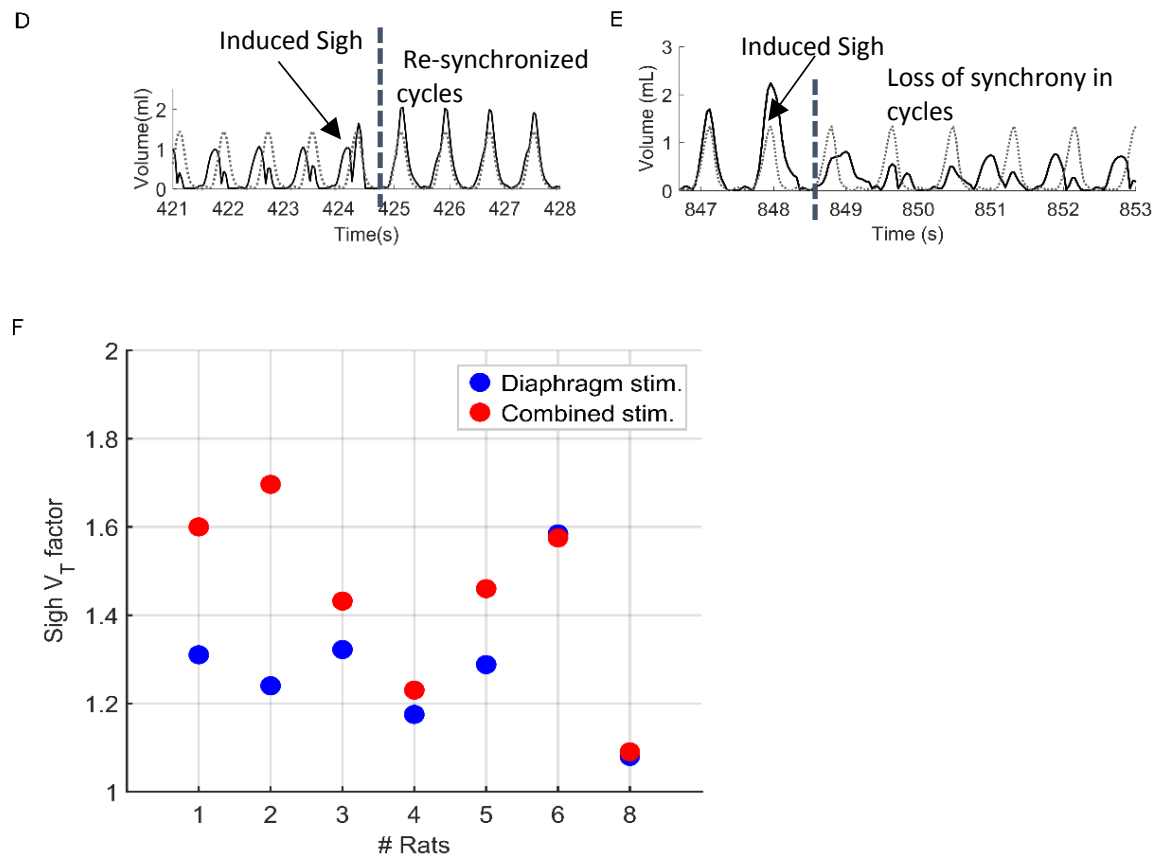

Figure 4: Different sighing phenomena and performance for sigh trials for only diaphragm muscle stimulation and combined muscle stimulation.

A Shows a sigh cycle in intrinsic breathing overlapped with a regular breathing cycle B, C shows induced breathing by only diaphragm stimulation and combined muscle stimulation respectively. D Shows an example of sigh functioning as a re-setter to restore synchrony between desired breathing and induced breathing. E Represents an example where after a sigh cycle, breathing lost synchrony. $\mathbf{F}$ Average tidal volume factors for sigh in only diaphragm muscle stimulation and combined muscle stimulation for all the animal. $\mathbf{G}$ Demonstrates a situation of spontaneous sigh occurrence before the stimulation for sigh. The vertical dashed line shows the time when the stimulation for sighs was provided. Though stimulation was provided for eliciting sigh, no response of larger tidal volume was visible. 
CHAPTER 5: DISCUSSION \& CONCLUSION 


\subsection{Discussion:}

This investigation presents the viability of a novel approach of combined muscle stimulation with the adaptive closed-loop controller. We showed the validation of attaining targeted breath volume in intact rats both for combined muscle stimulation and only diaphragm muscle stimulation. Efficiency of attaining the breath volume in terms of stimulation sent to the muscle is crucial. As in most of the functional electrical stimulation paradigm for maintaining the functional level, stimulation needs to be increased in order to offset the stimulation-induced fatigue. We performed both combined muscle stimulation and only diaphragm muscle stimulation by the adaptive closed-loop controller to compare fatigability of the diaphragm muscle in both cases. We calculated fatigue index for both trials by taking average of 50 cycles in initial and later parts of the trials. The fatigue index of the diaphragm muscle calculated during the lengthy trials of stimulation was significantly lower during combined muscle stimulation. Thus, this approach could be used to reduce stimulation-induced fatigue of the diaphragm muscle. Currently, commercially available Atrostim phrenic nerve pacing system uses four pole stimulation electrode. The quadripolar electrode keeps contact with the nerve evenly spaced way and activates the phrenic nerve or the diaphragm muscle sequentially, and thus reduce stimulation-induced fatigue (Ragnarsson 2007). Another commercially available phrenic nerve pacing system, the Medlmplant uses "carousel" stimulation method, where four electrodes are implanted in the phrenic nerve. The sequential activation of each electrode also works toward reducing the diaphragm muscle fatigue (Ragnarsson 2007). There are other ways to reduce the stimulation-induced fatigue such as varying pulse amplitude and 
frequency (Chou, Kesar, and Binder-Macleod 2008). In our experiments, only stimulation amplitude was varied by the adaption of PS control unit. Additionally, one possible limitation of the approach to measure fatiguability of the diaphragm muscle could be considering 50 cycles at the beginning and the later part of the trials. Because, considering 50 cycles for the fatigue index calculation might not be adequate, as loss of synchrony might make the average of charge delivered per cycle higher for some cases.

The other aim of our investigation was to assess the closed-loop controller's ability to generate sigh-like behavior by combined muscle stimulation and only diaphragm muscle stimulation. One tailed paired sample t-test suggested that induced tidal volume was significantly higher $(P<0.05)$ in combined muscle stimulation than only diaphragm muscle stimulation. The result suggests that combined muscle stimulation is a better approach to induce sigh volume than only diaphragm muscle stimulation. Currently, commercially available phrenic nerve pacing systems can also be used to provide sigh breaths by intermittent increase of stimulus frequency (DiMarco 2009). However, since all the phrenic nerve pacing systems are openloop system, adjustment of stimulus parameter is needed for inducing sighs. Whereas, our approach provided cyclic increase in stimulation amplitude to elicit large tidal volume without need of human intervention.

In our investigation, we observed sighing can improve the alignment of following targeted trajectory. However, we observed post sigh apnea in the experiments with intact rats. For individuals with a degree of breathing capacity, the adaptation could 
be paused for longer time (3-5 cycles) to recover from the post sigh apnea to better follow the desired trajectory after sighs. Another incident observed during sighing was the inability of the animal to induce sighing if intrinsic sigh happens few cycles before the stimulation sent out for a sigh. It could possibly be the stimulation was sent within the refractory period of the diaphragm muscle. As the external stimulation was provided during the time period, there was no response to the stimulus. To address this, we might develop an algorithm in the PS unit to detect spontaneous sighs few cycles before the presumed induced sighs and deliver the stimulation some cycles (5-10 cycles) later.

5.2 Limitations: In our investigation the model was spontaneously breathing intact rat under general anesthesia. But the current model will have little to no spinal reflex activity and reduced motor movement following $\mathrm{SCl}$. Though an intact model is widely used in respiratory FES testing, not considering an injured model is one of the limitations of this current method. Though the adaptation of stimulation parameters is achieved by the closed loop controller, inaccurate mapping of electrode implantation leads to increased charge delivery and decreased efficiency of the stimulation. Consequently, rapid muscle fatigue might occur. We considered visual inspection for evaluating twitch threshold, which couldn't be all the time flawless.

5.3 Conclusion: Respiratory muscle pacing is appearing as new promising option for ventilator-dependent $\mathrm{SCl}$ people. In patients with functional phrenic nerve, combined external intercostal and diaphragm pacing might maintain full-time 
ventilator support. Besides in individuals with partial phrenic nerve lesion, the external intercostal can support by supplementary inspiration. In animal experiments, combined intercostal and diaphragm pacing offered significant advantages compared to diaphragm pacing alone. Besides, activation of both the intercostal and diaphragm muscle synchronously could induce large tidal volume or sigh-like behavior, which might prevent progressive alveolar collapse. Further studies are needed to induce larger tidal volume for mimicking close to intrinsic sighs. Clinical trials with human patients might help to present challenges and further develop the technique. The broader aspect of human studies is needed to validate the benefit of this novel technique. 


\section{REFERENCE}

Abbas, J J, and H J Chizeck. 1995. "Neural Network Control of Functional Neuromuscular Stimulation Systems: Computer Simulation Studies." IEEE Transactions on Biomedical Engineering 42(11): 1117-27.

Alilain, Warren J et al. 2011. "Functional Regeneration of Respiratory Pathways after Spinal Cord Injury." Nature 475: 196. https://doi.org/10.1038/nature10199.

Berlly, Michael, and Kazuko Shem. 2007. "Respiratory Management During the First Five Days After Spinal Cord Injury." The Journal of Spinal Cord Medicine 30(4): 309-18.

Brown, Robert, Anthony F DiMarco, Jeannette D Hoit, and Eric Garshick. 2006. "Respiratory Dysfunction and Management in Spinal Cord Injury." Respiratory care 51(8): 853-70.

Butler, Jane E. 2007. "Drive to the Human Respiratory Muscles." Respiratory physiology \& neurobiology 159(2): 115-26.

Chou, Li-Wei, Trisha M Kesar, and Stuart A Binder-Macleod. 2008. "Using Customized Rate-Coding and Recruitment Strategies to Maintain Forces during Repetitive Activation of Human Muscles." Physical therapy 88(3): 363-75.

DiMarco, Anthony F. 2005. "Restoration of Respiratory Muscle Function Following Spinal Cord Injury: Review of Electrical and Magnetic Stimulation Techniques." Respiratory Physiology and Neurobiology 147(2-3 SPEC. ISS.): $273-87$.

. 2009. "Phrenic Nerve Stimulation in Patients with Spinal Cord Injury." Respiratory Physiology and Neurobiology.

De Troyer, André, Peter A Kirkwood, and Theodore A Wilson. 2005. "Respiratory Action of the Intercostal Muscles." Physiological reviews 85(2): 717-56. http://physrev.physiology.org/cgi/doi/10.1152/physrev.00007.2004.

Dimarco, Anthony F., K Budzinska, and Gerald S. Supinski. 1989. "Artificial Ventilation by Means of Electrical Activation of the Intercostal/Accessory Muscles Alone in Anesthetized Dogs." The American review of respiratory disease 139(4): 961-67.

DiMarco, Anthony F. 2001. "Neural Prostheses in the Respiratory System." Journal of rehabilitation research and development 38(6): 601-8.

Fairchild, Mallika D et al. 2010. "Repetetive Hindlimb Movement Using Intermittent Adaptive Neuromuscular Electrical Stimulation in an Incomplete Spinal Cord Injury Rodent Model." Experimental neurology 223(2): 623-33.

Fang, Z -., and J T Mortimer. 1991. "A Method to Effect Physiological Recruitment Order in Electrically Activated Muscle." IEEE Transactions on Biomedical Engineering 38(2): 175-79.

Galeiras Vazquez, Rita et al. 2013. "Respiratory Management in the Patient with Spinal Cord Injury." BioMed research international 2013: 168757. 
Jarosz, Renata, Meagan Littlepage, Graham Creasey, and Stephen McKenna. 2012. "Functional Electrical Stimulation in Spinal Cord Injury Respiratory Care." Topics in spinal cord injury rehabilitation 18(4): 315-21.

Kelly, B. J., and J. M. Luce. 1991. "The Diagnosis and Management of Neuromuscular Diseases Causing Respiratory Failure." Chest 99(6): 148594.

Kelly, Brian J, and John M Luce. 1991. "The Diagnosis and Management of Neuromuscular Diseases Causing Respiratory Failure." CHEST 99(6): 148594. https://doi.org/10.1378/chest.99.6.1485.

Levine, Sanford et al. 2008. "Rapid Disuse Atrophy of Diaphragm Fibers in Mechanically Ventilated Humans." New England Journal of Medicine 358(13): 1327-35. https://doi.org/10.1056/NEJMoa070447.

Li, Peng, and Kevin Yackle. 2017. "Sighing." Current Biology.

Levine, Sanford et al. 2008. "Rapid Disuse Atrophy of Diaphragm Fibers in Mechanically Ventilated Humans." New England Journal of Medicine 358(13): 1327-35.

Lumb, Andrew B. 2016. Nunn's Applied Respiratory Physiology EBook. Elsevier Health Sciences.

Mantilla, Carlos B., and Gary C. Sieck. 2011. "Phrenic Motor Unit Recruitment during Ventilatory and Non-Ventilatory Behaviors." Respiratory Physiology and Neurobiology.

Mauri, Tommaso et al. 2015. "Effects of Sigh on Regional Lung Strain and Ventilation Heterogeneity in Acute Respiratory Failure Patients Undergoing Assisted Mechanical Ventilation*." Critical Care Medicine 43(9).

Naik, Bhiken I, Carl Lynch, and Charles G Durbin. 2015. "Variability in Mechanical Ventilation: What's All the Noise About?" Respiratory care 60(8): 1203-10. http://www.ncbi.nlm.nih.gov/pubmed/25691765.

Patroniti M.D., Nicoló et al. 2002. "Sigh Improves Gas Exchange and Lung Volume in Patients with Acute Respiratory Distress Syndrome Undergoing Pressure Support Ventilation." Anesthesiology: The Journal of the American Society of Anesthesiologists 96(4): 788-94. https://doi.org/.

Pelosi, Paolo et al. 2003. "Sigh in Supine and Prone Position during Acute Respiratory Distress Syndrome." American Journal of Respiratory and Critical Care Medicine 167(4): 521-27. https://doi.org/10.1164/rccm.2002031980C.

Ragnarsson, K T. 2007. "Functional Electrical Stimulation after Spinal Cord Injury: Current Use, Therapeutic Effects and Future Directions." Spinal Cord 46: 255. https://doi.org/10.1038/sj.sc.3102091. 
Ratnovsky, Anat, David Elad, and Pinchas Halpern. 2008. "Mechanics of Respiratory Muscles." Respiratory Physiology \& Neurobiology 163(1-3): 8289. http://www.ncbi.nlm.nih.gov/pubmed/1592724.

Sieck, Gary C., and LF Ferreira. 2013. "Mechanical Properties of Respiratory Muscles." Comprehensive ... 3(4): 1553-67.

Siu, Ricardo et al. 2018. "Restoring Ventilatory Control Using an Adaptive Bioelectronic System." bioRxiv: 488577. http://biorxiv.org/content/early/2018/12/07/488577.abstract.

Yamauchi, Motoo et al. 2008. "Post-Sigh Breathing Behavior and Spontaneous Pauses in the C57BL/6J (B6) Mouse." Respiratory Physiology \& Neurobiology 162(2): 117-25. https://www.sciencedirect.com/science/article/pii/S1569904808001298 (March 17, 2019).

Walter, James S. et al. 2017. "Stimulation of Abdominal and Upper Thoracic Muscles with Surface Electrodes for Respiration and Cough: Acute Studies in Adult Canines." Journal of Spinal Cord Medicine 00(0): 1-11.

Walter, James S., Robert D. Wurster, Qianlong Zhu, and Franco Laghi. 2011. "Respiratory Muscle Pacing with Chronically Implanted Intramuscular Permaloc Electrodes: A Feasibility Study." The Journal of Rehabilitation Research and Development 48(2): 103. 